\title{
Oxygen tolerant RAFT polymerisation initiated by living bacteria
}

\author{
Mechelle R. Bennett ${ }^{\ddagger},{ }^{a}$ Cara Moloney ${ }^{\ddagger},{ }^{b}$ Francesco Catrambone ${ }^{\S},{ }^{c}$ Federico Turco $\$$, ${ }^{d}$ Katalin Kovács, ${ }^{e}$ Phillip J. Hill, ${ }^{\ddagger}$ \\ Cameron Alexander, e Frankie J. Rawson*, a and Pratik Gurnani*e \\ a. Division of Regenerative Medicine and Cellular Therapies, School of Pharmacy, University of Nottingham, University Park Campus, Nottingham NG7 2 \\ $R D(U K)$. \\ b. School of Medicine, BioDiscovery Institute, University of Nottingham, University Park Campus, Nottingham NG7 2RD (UK). \\ c. School of Life Sciences, BioDiscovery Institute, University of Nottingham, University Park Campus, Nottingham NG7 2RD (UK). \\ d. School of Pharmacy, BioDiscovery Institute, University of Nottingham, University Park Campus, Nottingham NG7 2RD (UK). \\ e. Division of Molecular Therapuetics, School of Pharmacy, University of Nottingham, University Park Campus, Nottingham NG7 $2 R D$ (UK). \\ f. Division of Microbiology, Brewing and Biotechnology, School of Biosciences, University of Nottingham, Sutton Bonington Campus, Nottingham LE12 \\ $5 R D(U K)$. \\ ¥Authors contributed equally, joint first author; §Authors contributed equally, joint second author; * Corresponding author \\ frankie.rawson@nottingham.ac.uk; pratik.gurnani@nottingham.ac.uk
}

Living organisms can synthesize a wide range of macromolecules from a small set of natural building blocks, yet there is potential for even greater materials diversity by exploiting biochemical processes to convert unnatural feedstocks into new abiotic polymers. Ultimately the synthesis of these polymers in situ might aid the coupling of organisms with synthetic matrices, and the generation of biohybrids or engineered living materials. The key step in biohybrid materials preparation is to harness the relevant biological pathways to produce synthetic polymers with predictable molar masses and defined architectures under ambient conditions. Accordingly, we report an aqueous, oxygen-tolerant RAFT polymerization platform based on a modified Fenton reaction which is initiated by Cupriavidus metallidurans $\mathrm{CH} 34$, a bacterial species with iron reducing capabilities. We show the synthesis of a range of water-soluble polymers under normoxic conditions, with control over the molar mass distribution, and also the production of block copolymer nanoparticles via polymerization-induced selfassembly. Finally, we highlight the benefits of using a bacterial initiation system by recycling the cells for multiple polymerisations. Overall, our method represents a highly versatile approach to producing well-defined polymeric materials within a hybrid natural-synthetic polymerization platform and in engineered living materials with properties beyond those of biotic macromolecules.

\section{Introduction}

Nature exploits a vast array of biological pathways to produce biotic macromolecules (polysaccharides, proteins, DNA, RNA etc.) derived from a small subset of monomers (e.g. sugars, amino acids, nucleobases etc.). In contrast, the chemical industry has made available an enormous stock of monomers, particularly those with reactive double bonds, to provide routes to an almost limitless set of abiotic macromolecules. Polymers derived from vinylic or acrylic functionality have found use in biomedicine, ${ }^{1,2}$ and as energy, ${ }^{3}$ and information storage materials. ${ }^{4,5}$ Combining biosynthetic pathways with abiotic monomers could therefore generate an even greater diversity of materials and, if conducted in the presence of an organism with appropriate biochemical functionality, allow hybrid synthetic/natural interfaces and engineered living materials (ELMs) to be formed.

Cellular metabolism is underpinned by electron transport via redox pathways. We and others have shown that these pathways can be used in cell-activated polymerization. ${ }^{6-11}$ Prior reports have focused on the metal reducing activity of bacteria (e.g. E. coli, C. metallidurans, S. oneidensis) to mediate the active and dormant states of copper, iron and other metallic catalysts for atom transfer radical polymerizations (ATRP). ${ }^{6-9,11}$ However, ATRP suffers a disadvantage of requiring careful tuning of the concentrations of bacteria and metal complexes to control the balance of growing and dormant chains for desirable kinetics and molar mass distribution. ${ }^{12}$ In contrast, RAFT polymerization, which is a chain-transfer agent mediated polymerization, requires instead a constant flux of external radicals. In many biological environments, a source of radicals is readily available, thus 
RAFT might be inherently easier to control than cell instructed ATRP, which is adversely affected by alternate indirect initiation pathways from bacterial cultures. ${ }^{13}$

Whilst it has been shown that the generic reducing environment of bacteria can be used to produce organic radicals from the reduction of an aryl diazonium salt, which initiates the RAFT process, ${ }^{10}$ this has been achieved so far only under anoxic conditions, hindering translation to biological applications. Conversely, many oxygen tolerant RAFT polymerisations have been reported, ${ }^{14}$ either by polymerizing directly through oxygen ${ }^{15-17}$ or utilising a scavenger such as an enzyme ${ }^{18-20}$ or oxygen trap ${ }^{21-25}$, which has enabled ultralow reaction volumes, ${ }^{17}$, 19, 22 3D/4D printing ${ }^{21,} 26$ and high throughput platforms, ${ }^{22}$ but to the best of our knowledge have not been applied in a bacterially initiated RAFT polymerisation.

Accordingly, in this study, we present a new oxygen tolerant bacteria-initiated RAFT polymerization, by utilizing an adapted Fenton polymerisation. ${ }^{27,28}$ Ourapproach harnesses the substantially faster reaction rate (4-5 orders of magnitude) between hydrogen peroxide and $\mathrm{Fe}^{2+}$ than with $\mathrm{Fe}^{3+}$ to produce hydroxyl radicals to mediate the RAFT process. While a typical Fenton polymerization procedure directly implements $\mathrm{Fe}^{2+}$ to avoid this, we postulated that we could use the $\mathrm{Fe}^{3+}$ reducing capabilities of $\mathrm{C}$. metallidurans $\mathrm{CH} 34$ metabolism, which instructs the in situ formation of $\mathrm{Fe}^{2+}$, and accelerate the formation of hydroxyl radicals to initiate the RAFT process. To achieve oxygen tolerance, we were inspired by previous studies which utilized glucose oxidase (GOx) to deoxygenate transiently the reaction media from a glucose feedstock. ${ }^{18,19}$ This approach provided a dual benefit, as a key byproduct from GOx deoxygenation is hydrogen peroxide which could be fed into our bacterially instructed Fenton reaction (Scheme 1). ${ }^{30}$ Using this approach, we report the optimization and mechanistic evaluation of our bacterially mediated Fenton polymerisation. We highlight this through the synthesis of a range of well-defined RAFT polymers and polymer nanoparticles in open-to-air vessels under aqueous conditions.

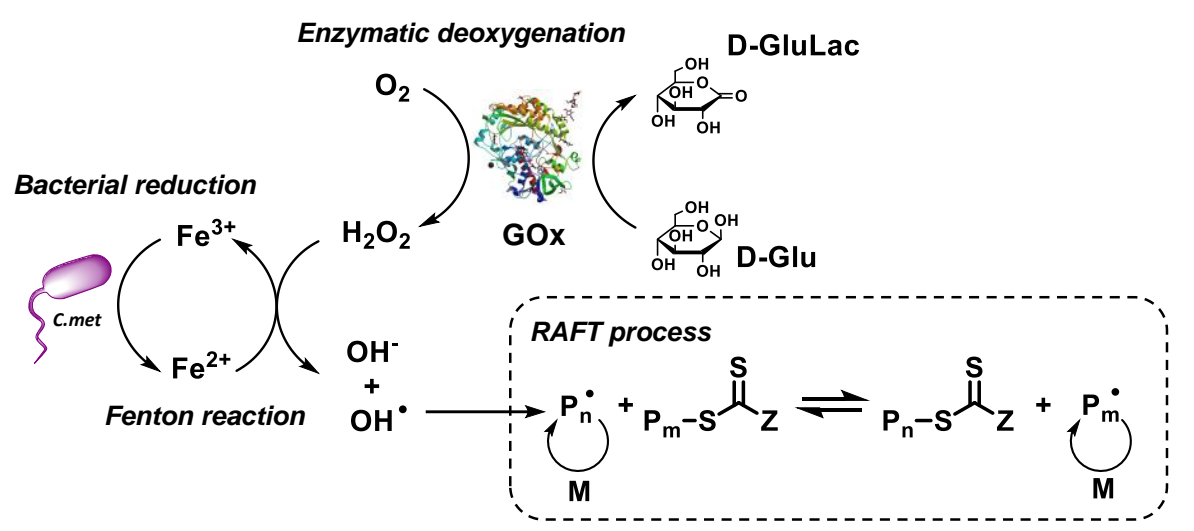

Scheme 1. Fenton GOx RAFT process initiated by reducing agents: ascorbic acid (AscA) or bacteria. D-Glucose (DG) is converted to D-Glucanolactate (DGA) by glucose oxidase (GOx) which consumes $\mathrm{O}_{2}$ in the process to form $\mathrm{H}_{2} \mathrm{O}_{2}$. Without the presence of reducing agents, polymerisation should not take place. GOx protein image from PDB ID: 3QVP.A. ${ }^{29}$

\section{Results and Discussion}

Before conducting our bacteria mediated Fenton RAFT polymerisations, we initially evaluated the viability of $C$. metallidurans $\mathrm{CH} 34$ cells in the presence of a range of water-soluble monomers to ensure any observable polymerization was not caused by cell lysis (Figure S1 and Table S1). Both N,N-dimethylacrylamide (DMA) and $\mathrm{N}$-hydroxyethylacrylamide (HEA) exhibited an $\mathrm{MIC}_{50}$ above $100 \mathrm{mM}$. However, $\mathrm{N}$-acryloyl morpholine (NAM) displayed some toxicity towards the bacterial cultures ( $\mathrm{MIC}_{50}=42.5 \mathrm{mM}$ ). As a result of this, a concentration of 25 mM NAM was employed as this ensured c. 70\% bacterial viability, a similar viability was observed at a monomer concentration of $100 \mathrm{mM}$ for DMA and HEA. 
To test our bacteria instructed Fenton-RAFT hypothesis, we incubated a mixture of DMA monomer, carboxyethyl propanoic acid trithiocarbonate (CEPTC) water soluble RAFT agent, $\mathrm{FeCl}_{3}$ as the $\mathrm{Fe}^{3+}$ source, glucose oxidase and glucose with a $C$. metallidurans culture $\left(1.7 \times 10^{10}\right.$ colony forming units $\left.(\mathrm{CFU}) \mathrm{mL}^{-1}\right)$ in phosphate buffered saline (PBS) ([DMA]:[CTA]:[ $\left[\mathrm{FeCl}_{3}\right]:[\mathrm{GOx}]:[\mathrm{Glucose}]=200: 1: 5.3: 0.002: 0.8$ ) and heated the suspension to $30^{\circ} \mathrm{C}$ in an open to air vessel under normoxic conditions for $24 \mathrm{~h}$. Aside from its iron reducing properties, $C$. metallidurans lacks the glucose transporter, thus we deemed it unlikely that the bacterial cells were reducing the glucose concentration through metabolization. ${ }^{31}$ Conducting the polymerisations in PBS instead of growth medium also mitigated the risk of incorporating additional reducing agents which may contribute to redox based radical initiation pathways. After removal of the bacteria and iron oxide precipitate, ${ }^{1} \mathrm{H}$ NMR spectroscopy confirmed the presence of polymer, with monomer conversion reaching 53\% (Figure 1a). SEC analysis indicated a monomodal molecular weight distribution with low dispersity $(\theta=1.12)$ and low molar mass $\left(M_{\mathrm{n}, \mathrm{SEC}}=19,900 \mathrm{~g}\right.$ $\mathrm{mol}^{-1}$ ) as is expected for RAFT polymerisation. Crucially, control experiments omitting $\mathrm{FeCl}_{3}$ or with $C$. metallidurans cultures which were heat killed $\left(3.6 \times 10^{2} \mathrm{CFU} \mathrm{mL}^{-1}\right)$ displayed no monomer conversion indicating the importance of metabolically active cells for successful polymerization (Table S2). Noticeably, reaction mixtures containing $\mathrm{FeCl}_{3}$ but in the absence of bacteria yielded a small level of polymerization (10\% monomer conversion) which we suspect is due to the slower $\mathrm{Fe}^{3+}$ mediated Fenton reaction, producing a low concentration of hydroxyl radicals which still contribute to conversion (Figure 1b, Figure S2. Polymerisations in the absence of CTA yielded substantially higher molar masses $\left(M_{\mathrm{n}, \mathrm{SEC}}=451,000 \mathrm{~g} \mathrm{~mol}^{-1}\right)$ and high dispersity $(\theta=2.11)$ following a conventional free radical mechanism (Figure 1c).
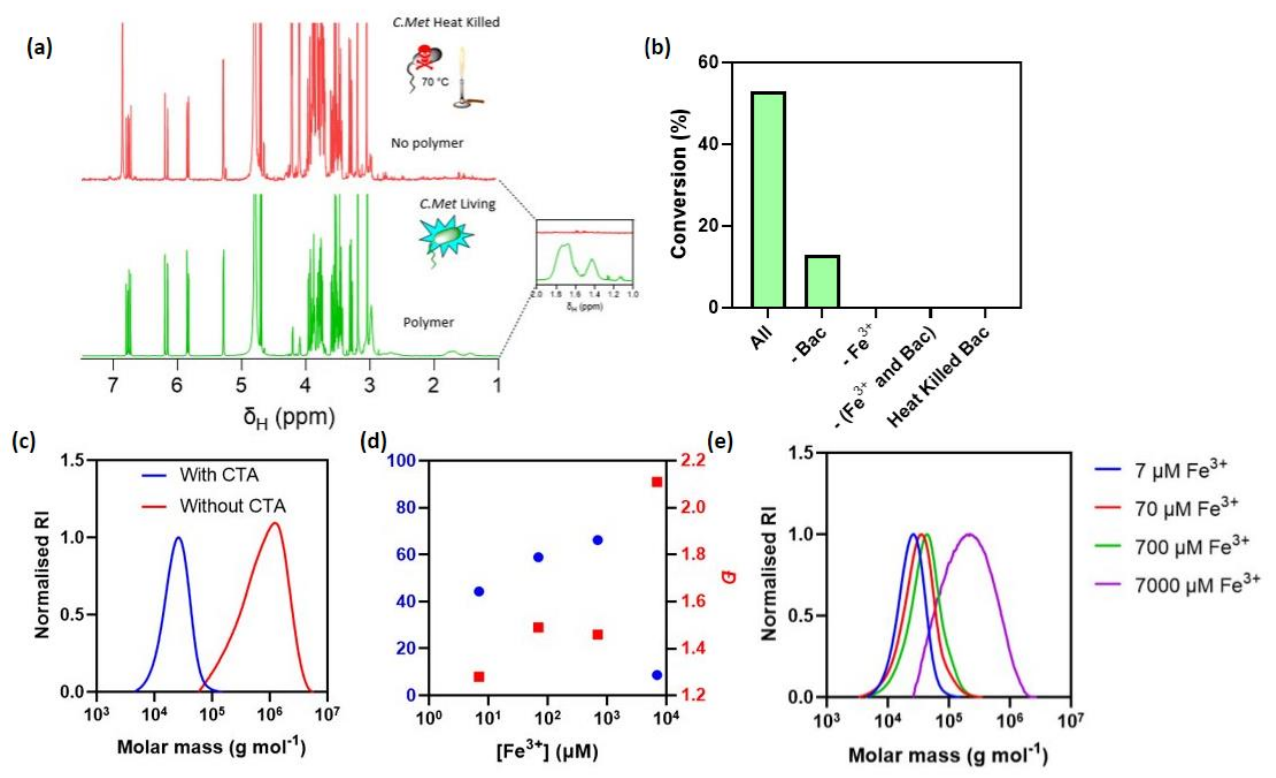

Figure 1 (a) ${ }^{1} \mathrm{H}$ NMR stacked spectra of bacterial initiated polymerisations of DMA in air at $30^{\circ} \mathrm{C}$ with either living $\mathrm{C}$. metallidurans (bottom, green) and heat killed C. metallidurans (top, red). (b) Conversion as calculated by ${ }^{1} \mathrm{H} N M R(400 \mathrm{MHz}$, $\mathrm{D}_{2} \mathrm{O}$ ) of final time point (20 hours) in bacterial initiated polymerisations showing the need for live bacteria and a Fe ${ }^{3+}$ source for high conversion polymerisation to occur. (c) SEC (DMF) overlay of polymers produced with and without the addition of CTA. (d) Effect of concentration of $\mathrm{Fe}^{3+}$ on conversion from ${ }^{1} \mathrm{H} \mathrm{NMR}\left(400 \mathrm{MHz}, \mathrm{D}_{2} \mathrm{O}\right)$ and $\bigoplus$ from SEC (DMF). (e) Corresponding SEC (DMF, RI detector).

When hydroxyl radicals are generated from the bacterially produced $\mathrm{Fe}^{2+}, \mathrm{Fe}^{3+}$ is regenerated during the Fenton reaction. We, therefore, postulated that the bacteria could recycle the available $\mathrm{Fe}^{3+}$ for further Fenton polymerisations at a reduced $\mathrm{FeCl}_{3}$ concentration. Accordingly, the polyDMA produced in polymerisations conducted at $7 \mu \mathrm{M}$ maintained narrow dispersities $(\theta \sim 1.28$, Figure $1 \mathrm{~d})$ and still achieved moderate monomer conversions (44\%). Therewas an increasing trend correlating $\mathrm{FeCl}_{3}$ concentration with monomer conversion between 7 and $700 \mu \mathrm{M}$, reaching a maximum of $66.2 \%$, also resulting in an increase in $\oslash$ from 1.28 to 1.49. All 
polymers had unimodal molar mass distributions with similar $M_{n, S E C}$ to their $M_{n, t h}$ values (Figure 1e). Strikingly at $7 \mathrm{mM}$ we observed a substantial reduction in monomer conversion to $9 \%$, much broader molar mass distributions $(\theta=2.11)$ and $M_{\mathrm{n}, \mathrm{SEC}} 50$-fold higher than the $M_{\mathrm{n}, \mathrm{h}}$ which is more consistent with free radical polymerization likely caused by excess oxidation of free RAFT agent and possible toxicity towards $C$. metallidurans. For this reason, we adopted Fe concentrations of $7 \mu \mathrm{M}$ for the remaining experiments.

Bacteria assisted Fenton RAFT polymerizations with HEA and NAM (conducted at $100 \mathrm{mM}$ and $25 \mathrm{mM}$ monomer solutions respectively) displayed similar monomer conversions to DMA (37 and 40\% respectively), albeit with higher dispersities ( $\Theta \sim 1.6$ for both polymerisations, compared to 1.28 for DMA) (Figure 2a,Table S3, Figure S3). Although HEA polymers displayed moderately similar experimental and theoretical molar masses, the NAM analogues were 10-fold higher in molar mass than expected, attributed either due to the difference in monomer concentration or the poorer cell tolerability described above. To probe this, we performed a copolymerization produced a copolymer with similar experimental and theoretical molar masses and low dispersity $(\theta=1.21)$, suggesting this was due to the overall monomer concentration not NAM toxicity.

(a)

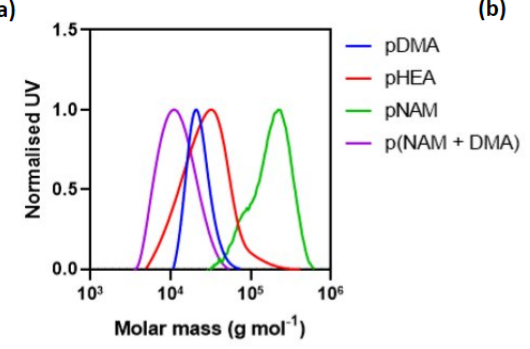

(d)

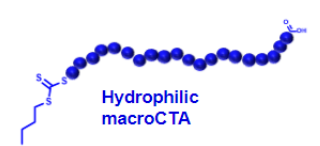

(b)
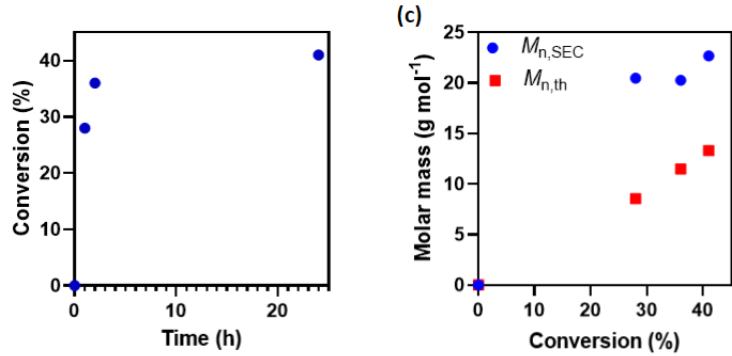

(e)

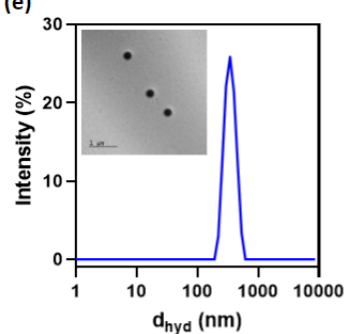

Figure 2. (a) SEC (aqueous, UV detector) of polymers prepared with varying monomers. (b) ${ }^{1} \mathrm{H} N M R\left(400 \mathrm{MHz}, \mathrm{D}_{2} \mathrm{O}\right)$ kinetic plot showing the effect of polymerisation time on the conversion. (c) Comparison of $M_{n, S E C}$ (aqueous, $U V$ signal) and $M_{n, t h}$ as a function of conversion calculated by ${ }^{1} \mathrm{H}$ NMR $\left(400 \mathrm{MHz}, \mathrm{D}_{2} \mathrm{O}\right)$. (d) Schematic representation of bacteria-initiated polymerization-induced self-assembly to form spherical polymer nanoparticles. (e) Recorded DLS data for nanoparticles formed by PISA ( $d_{\text {hyd }}=456 \mathrm{~nm}$ ); Inset - representative TEM image.

We then investigated the polymerization kinetics of our bacteria-initiated RAFT polymerisation by sampling a DMA polymerization at $1 \mathrm{~h}, 2 \mathrm{~h}$ and $24 \mathrm{~h}$, monitoring monomer conversion and $M_{\mathrm{n}, S E C}$. Notably, we observed the polymerization did not proceed above $41 \%$ monomer conversion under these conditions (Figure $2 \mathrm{~b}$ ). This conversion is in line with other bacterial radical polymerization systems, $7,8,10$ and we anticipate is due to the low initial monomer conversion, which quickly depletes retarding the ensuing polymerization reaction, compounded by the consumption of the glucose feedstock by GOx. Although a uniform molar mass distribution $(\theta<1.40)$ and retention of the trithiocarbonate was observed across all time points indicating contribution by the chain transfer agent (Figure S4b), only partial linear evolution between $M_{n, S E C}$ and monomer conversion for RAFT polymerizations was observed, suggesting some RAFT characteristics.(Table S3 and (Figure 2c). This is supported by the first order kinetic plot (Figure S4), which indicates a fast linear reaction between 0 and $2 \mathrm{~h}$, which then reached a plateau in terms of rats after $35 \%$ monomer conversion (Figure S4a). Although the relatively low monomer conversion of this polymerization is a potential limitation, the necessity for active metabolism and 
living cells to initiate polymerization, a notable difference compared to previous strategies, ${ }^{10}$ means conversion is correlated to the tolerability of the chosen monomers.

One of the major advantages of RAFT polymerisations is the ability to prepare block copolymer nanoparticles with relative ease, ${ }^{32}$ which have enormous potential in drug delivery ${ }^{33}$ and other applications. ${ }^{34}$ An extremely versatile route that has been explored for the last decade is the polymerization-induced self-assembly (PISA), enabling the preparation of well-defined nanoparticles in situ during the polymerization which can be conducted under completely aqueous conditions (Figure $2 \mathrm{~d}$ ). ${ }^{35,36}$ Given the success of thisapproach and our encouraging results with bacteria-initiated solution polymerisations, we explored if we could utilize the methodology presented here to produce block copolymer nanoparticles via PISA. The pDMA 75 mCTA was extended with a target 200 units of diacetone acrylamide, a monomer known to undergo PISA, ${ }^{37-39}$ reaching quantitative monomer conversion as is expected in PISA due to the high local monomer concentration within the growing particles. Particle size analysis via both DLS and TEM indicates successful nanoparticle preparation with corroborative sizes between the two techniques (Figure 2e). However, due to the low concentrations used in our PISA reaction no molar mass information could be obtained from dried particles. The ability to produce nanoparticles using this system could in future offer the potential of biomimetic extracellular vesicles, which are achievable through PISA ${ }^{40}$ which could for instance transport innate quorum sensing molecules. ${ }^{41}$

One of the major advantages of utilising living systems to initiate chemical reactions or indeed polymerisations is their ability to be reused or expanded through culture to remove feedstock requirements, important for the sustainability of these processes. Hence, we subsequently investigated if the initial $C$. metallidurans culture could be recycled for several polymerization reactions by pelleting the cells through centrifugation and resuspension with a new polymerization mixture (Figure 3a). It was found that the initial bacterial culture could be reused at least three times using without supplementing with growth media or nutrients. Interestingly the monomer conversion and $M_{\mathrm{n}, \mathrm{SEC}}$ was variable between each cycle at 40,80 and $50 \%$ for the three consecutive polymerisations and 18,800,32,500 and $26,500 \mathrm{~g} \mathrm{~mol}^{-1}$ respectively, each with low $(\theta \sim 1.3)$ in all cases. While further investigation is required to understand fully these differences, we anticipate that some bacterial proliferation or changes in bacterial metabolism may affect final conversion. (Figure 3b, Figure 3c). A similar phenomenon was reported by Keitz and co-workers for the bacteria mediated $\mathrm{Cu}(\mathrm{I})$-catalysed azide-alkyne cycloaddition, where subsequent cycles yielded different reactionconversions to the first cycle, which they suggested was due to bacterial growth between cycles 1 and $2 .{ }^{42}$
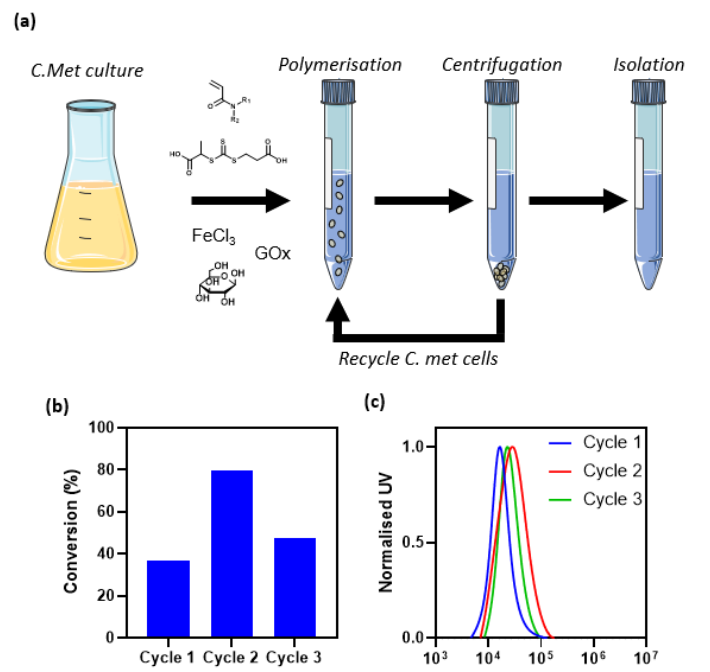

(c)

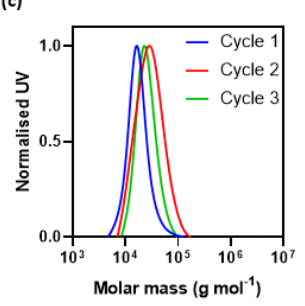

Figure 3. (a) Schematic representation of bacteria recycling (b) ${ }^{1} \mathrm{H} N M R\left(400 \mathrm{MHz}, \mathrm{D}_{2} \mathrm{O}\right.$ ) conversion of polymerisations with bacteria recycling. (c) Corresponding SEC (aqueous, UV detector). 


\section{Conclusions}

In conclusion, we have developed an oxygen tolerant bacterially initiated polymerization method which can be used to produce macromolecules with defined length via RAFT polymerisation. To achieve this, we utilized the reducing capabilities of $\mathrm{C}$. metallidurans to produce $\mathrm{Fe} 2+$ in situ and a simultaneous glucose oxide catalysis pathway to generate hydrogen peroxide from a glucose feedstock, which then reacts to produce hydroxyl radicals and initiate polymerization. We found that high monomer conversion could only be achieved with actively metabolising bacteria and in the presence of $\mathrm{Fe} 3+$, supporting our proposed mechanism. Synthesized polymers exhibited the characteristics of conventional RAFT polymerisations such as narrow molecular weight distributions, retention of end-group fidelity and similar average molar masses, albeit with some limits in terms of blocking efficiencies. We exemplified this polymerization technique by utilising monomers known to undergo polymerization-induced self-assembly to produce bacterially synthesized polymer nanoparticles. Finally, we showcased the ability for the bacteria to be a reusable component for radical generation and thus polymerization. This microbial redox pathway to produce well defined polymers could open the potential for hybrid natural and non-natural material platforms and thus new engineered living materials.

\section{Acknowledgements}

The authors thank Dr Michael W. Fay of the Nanoscale and Microscale Research Centre (nmRC) for TEM imaging and technical assistance. This work was supported by the Engineering and Physical Sciences Research Council (EPSRC) (grant numbers [EP/R004072/ 1 EP/N03371X1, EP/L022494/1], the Biotechnology and Biological Sciences Research Council (grant number BB/L013940/1), and the Royal Society (Wolfson Research Merit Award WM150086 to C.A.). PG research is funded by the Department of Health and Social Care using UK Aid funding and is managed by the Engineering and Physical Sciences Research Council (EPSRC, grant number: EP/R013764/1). The views expressed in this publication are those of the author(s) and not necessarily those of the Department of Health and Social Care. We also thank Douglas Crackett and Paul Cooling for expert technical assistance and Carol Turrill for outstanding administrative support.

\section{References}

1. A. L. Hook, C.-Y. Chang, J. Yang, J. Luckett, A. Cockayne, S. Atkinson, Y. Mei, R. Bayston, D. J. Irvine, R. Langer, D. G. Anderson, P. Williams, M. C. Davies and M. R. Alexander, Nature Biotechnology, 2012, 30, 868-875.

2. A. Kuroki, P. Sangwan, Y. Qu, R. Peltier, C. Sanchez-Cano, J. Moat, C. G. Dowson, E. G. L. Williams, K. E. S. Locock, M. Hartlieb and S. Perrier, ACS Applied Materials \& Interfaces, 2017, 9, 40117-40126.

3. K.-A. Hansen and J. P. Blinco, Polymer Chemistry, 2018, 9, 1479-1516.

4. R. Szweda, M. Tschopp, O. Felix, G. Decher and J.-F. Lutz, Angewandte Chemie International Edition, 2018, 57, 15817-15821.

5. J. M. Lee, M. B. Koo, S. W. Lee, H. Lee, J. Kwon, Y. H. Shim, S. Y. Kim and K. T. Kim, Nature Communications, 2020, 11, 56.

6. M. R. Bennett, P. Gurnani, P. J. Hill, C. Alexander and F. J. Rawson, Angewandte Chemie International Edition, 2020, 59, 4750-4755.

7. G. Fan, C. M. Dundas, A. J. Graham, N. A. Lynd and B. K. Keitz, Proceedings of the National Academy of Sciences, 2018, 115, 4559-4564.

8. G. Fan, A. J. Graham, J. Kolli, N. A. Lynd and B. K. Keitz, Nature Chemistry, 2020, 12, 638-646.

9. E. P. Magennis, F. Fernandez-Trillo, C. Sui, S. G. Spain, D. J. Bradshaw, D. Churchley, G. Mantovani, K. Winzer and C. Alexander, Nature Materials, 2014, 13, 748-755.

10. M. D. Nothling, H. Cao, T. G. McKenzie, D. M. Hocking, R. A. Strugnell and G. G. Qiao, Journal of the American Chemical Society, 2021, 143, 286-293. 
11. Y. Luo, Y. Gu, R. Feng, J. Brash, Ahmed M. Eissa, D. M. Haddleton, G. Chen and H. Chen, Chemical Science, 2019, 10, 5251-5257.

12. M. Ouchi and M. Sawamoto, Macromolecules, 2017, 50, 2603-2614.

13. S. Perrier, Macromolecules, 2017, 50, 7433-7447.

14. J. Yeow, R. Chapman, A. J. Gormley and C. Boyer, Chemical Society Reviews, 2018, 47, 4357-4387.

15. G. Gody, R. Barbey, M. Danial and S. Perrier, Polymer Chemistry, 2015, 6, 1502-1511.

16. P. Gurnani, T. Floyd, J. Tanaka, C. Stubbs, D. Lester, C. Sanchez-Cano and S. Perrier, Polymer Chemistry, 2020, 11, 1230-1236.

17. J. Tanaka, P. Gurnani, A. B. Cook, S. Häkkinen, J. Zhang, J. Yang, A. Kerr, D. M. Haddleton, S. Perrier and P. Wilson, Polymer Chemistry, 2019, 10, 1186-1191.

18. R. Chapman, A. J. Gormley, K.-L. Herpoldt and M. M. Stevens, Macromolecules, 2014, 47, 8541-8547.

19. R. Chapman, A. J. Gormley, M. H. Stenzel and M. M. Stevens, Angewandte Chemie International Edition, 2016, 55, 4500-4503.

20. M. D. Nothling, Q. Fu, A. Reyhani, S. Allison-Logan, K. Jung, J. Zhu, M. Kamigaito, C. Boyer and G. G. Qiao, Advanced Science, 2020, 7, 2001656.

21. A. Bagheri, C. W. A. Bainbridge, K. E. Engel, G. G. Qiao, J. Xu, C. Boyer and J. Jin, ACS Applied Polymer Materials, 2020, 2, 782-790.

22. A. J. Gormley, J. Yeow, G. Ng, Ó. Conway, C. Boyer and R. Chapman, Angewandte Chemie International Edition, 2018, 57, 1557-1562.

23. G. Ng, J. Yeow, J. Xu and C. Boyer, Polymer Chemistry, 2017, 8, 2841-2851.

24. C. Wu, K. Jung, Y. Ma, W. Liu and C. Boyer, Nature Communications, 2021, 12, 478.

25. J. Xu, K. Jung and C. Boyer, Macromolecules, 2014, 47, 4217-4229.

26. Z. Zhang, N. Corrigan, A. Bagheri, J. Jin and C. Boyer, Angewandte Chemie International Edition, 2019, 58, 17954-17963.

27. A. Reyhani, T. G. McKenzie, Q. Fu and G. G. Qiao, Macromolecular Rapid Communications, 2019, 40, 1900220.

28. A. Reyhani, T. G. McKenzie, H. Ranji-Burachaloo, Q. Fu and G. G. Qiao, Chemistry - A European Journal, 2017, 23, 7221-7226.

29. P. R. Kommoju, Z. W. Chen, R. C. Bruckner, F. S. Mathews and M. S. Jorns, Biochemistry, 2011, 50, 55215534.

30. A. Reyhani, M. D. Nothling, H. Ranji-Burachaloo, T. G. McKenzie, Q. Fu, S. Tan, G. Bryant and G. G. Qiao, Angewandte Chemie International Edition, 2018, 57, 10288-10292.

31. R. Van Houdt, A. Provoost, A. Van Assche, N. Leys, B. Lievens, K. Mijnendonckx and P. Monsieurs, Genes, 2018, 9, 507.

32. D. J. Keddie, Chemical Society Reviews, 2014, 43, 496-505.

33. H. Cabral, K. Miyata, K. Osada and K. Kataoka, Chemical Reviews, 2018, 118, 6844-6892.

34. H. Feng, X. Lu, W. Wang, N.-G. Kang and J. W. Mays, Polymers, 2017, 9, 494.

35. N. J. Warren and S. P. Armes, Journal of the American Chemical Society, 2014, 136, 10174-10185.

36. N. J. W. Penfold, J. Yeow, C. Boyer and S. P. Armes, ACS Macro Letters, 2019, 8, 1029-1054.

37. J. He, Q. Xu, J. Tan and L. Zhang, Macromolecular Rapid Communications, 2019, 40, 1800296.

38. S. J. Byard, M. Williams, B. E. McKenzie, A. Blanazs and S. P. Armes, Macromolecules, 2017, 50, 14821493.

39. J. Y. Rho, G. M. Scheutz, S. Häkkinen, J. B. Garrison, Q. Song, J. Yang, R. Richardson, S. Perrier and B. S. Sumerlin, Polymer Chemistry, 2021, 12, 3947-3952.

40. L. D. Blackman, S. Varlas, M. C. Arno, A. Fayter, M. I. Gibson and R. K. O’Reilly, ACS Macro Letters, 2017, 6, 1263-1267.

41. M. Toyofuku, K. Morinaga, Y. Hashimoto, J. Uhl, H. Shimamura, H. Inaba, P. Schmitt-Kopplin, L. Eberl and N. Nomura, The ISME Journal, 2017, 11, 1504-1509. 
42. G. Partipilo, A. J. Graham, B. Belardi and B. K. Keitz, ACS Central Science, 2022, DOI: 10.1021/acscentsci.1c01208. 